\title{
Benchmark Solutions of Large Problems for Evaluating Accuracy and Efficiency of Electromagnetics Solvers
}

\author{
Özgür Ergül \\ Department of Mathematics and Statistics \\ University of Strathclyde, Glasgow, UK \\ Email: ozgur.ergul@strath.ac.uk
}

\author{
Levent Gürel ${ }^{1,2}$ \\ ${ }^{1}$ Department of Electrical and Electronics Engineering \\ ${ }^{2}$ Computational Electromagnetics Research Center (BiLCEM) \\ Bilkent University, Bilkent, Ankara, Turkey \\ Email: lgurel@bilkent.edu.tr
}

\begin{abstract}
We present a set of benchmark problems involving conducting spheres and their solutions using a parallel implementation of the multilevel fast multipole algorithm (MLFMA). Accuracy of the implementation is tested by comparing the computational results with analytical Mie-series solutions. Reference solutions are made available on an interactive website to evaluate and compare the accuracy and efficiency of fast solvers. We also demonstrate the capabilities of our solver on real-life problems involving complicated targets, such as the Flamme.
\end{abstract}

\section{INTRODUCTION}

Recently, solutions of large-scale electromagnetics problems have attracted great interest from the scientific community [1][12]. Implementations of fast algorithms, such as the multilevel fast multipole algorithm (MLFMA) [1], on parallel computers have enabled accurate solutions of real-life problems discretized with hundreds of millions of unknowns [9][12]. Recent achievements have been successfully employed to solve various problems involving complicated objects that are larger than $1000 \lambda$ [12], where $\lambda$ is the wavelength. As in other computational disciplines, there are two important aspects of large-scale electromagnetic simulations: accuracy and efficiency. Assessments of algorithms and their implementations should consider both aspects. Nevertheless, it is not trivial to determine a set of common rules for a fair comparison of solvers. Testing different implementations on different problems with diverse geometries also prevents direct comparisons.

In this study, we consider large-scale scattering problems involving perfectly conducting spheres that can be used for benchmarking fast electromagnetics solvers. We present fast and accurate solutions of these problems with a parallel implementation of MLFMA [8],[12]. We test the accuracy of our implementation by comparing numerical results with those obtained via analytical Mie-series solutions. We also make the reference Mie-series solutions available on an interactive website at

WWW.cem.bilkent.edu.tr/benchmark

that can be used by the members of the electromagnetics community to test the accuracy of their results. The website also provides numerical results of other scattering problems for benchmarking purposes, including a famous object, namely, the NASA Almond. All computational results are obtained with our parallel MLFMA solver. Solutions of similar problems can provide essential information for the comparison of different solvers.

This paper presents some of the benchmark solutions obtained by using a parallel implementation of MLFMA. We also discuss the accuracy and efficiency of solutions and demonstrate the capabilities of our solver on complicated problems involving the stealth airborne target Flamme.

\section{FAst and AcCurate Solutions with MLFMA}

First, we discuss the two aspects (accuracy and efficiency) of solutions, particularly in the context of MLFMA.

\section{A. Efficiency}

In order to measure the efficiency of an implementation, the most important quantity is the time required for the solution of problems. The computing time depends on the computer; but properties of the computer hardware, particularly the clock rate of the processor(s), can be provided with the computing time for a thorough assessment of the efficiency. For parallel implementations, parallelization efficiency and speedup with respect to the number of processors are also important parameters. However, parallelization efficiency (or speedup) may not be a good indicator for the actual efficiency of the implementation. In fact, as discussed in [8], parallelization results can be misleading without the computing time. For example, an implementation involving many on-the-fly calculations can be "embarrassingly" parallelizable, while it can be very slow and inefficient considering the computing time. In addition, low-complexity algorithms, such as MLFMA, are difficult to parallelize; but, as the problem size grows, their parallel implementations become much faster than perfectly parallel algorithms with higher complexities. Consequently, measuring the computing time remains as the golden standard of assessing the efficiency of fast algorithms. 


\section{B. Accuracy}

Efficiency alone is not meaningful without accuracy. This is because accuracy can be traded against computing time in solvers with controllable error. In the following, we list major error sources of MLFMA and describe how they can (but should not) be relaxed to "improve" the efficiency.

1) Discretization: Numerical results are drastically affected by the discretization. Unfortunately, this pre-processing step is usually omitted and not mentioned in many publications. For example, our numerical experiments show that, using the RaoWilton-Glisson (RWG) functions on planar triangles, the mesh size should not exceed $\lambda / 10$ to attain maximum $0.5 \%$ error in the far-zone fields. Denser triangulations and/or higherorder discretizations are needed for solutions with the secondkind integral equations, such as the magnetic-field integral equation (MFIE) and the combined-field integral equation (CFIE). Using coarse discretizations usually improves the efficiency, e.g., due to the increasing sparsity of the near-field interactions in MLFMA, but may significantly deteriorate the accuracy.

2) Integral Equation: Another parameter is the choice of the integral equation to formulate the problem. As mentioned above, the second-kind integral equations, such as MFIE and CFIE, may require denser discretizations compared to the first-kind integral equations, such as the electric-field integral equation (EFIE), for the same level of accuracy. In fact, the combination parameter $\alpha$ in CFIE $=\alpha$ EFIE $+(1-\alpha)$ MFIE directly affects the accuracy and efficiency. Lower values, such as $\alpha=0.2$, can be optimal in terms of iterative solutions (and efficiency), but may not be suitable for obtaining the desired level of accuracy.

3) Direct Interactions: Similar to some other fast solvers, MLFMA implementations involve near-zone interactions that cannot be factorized and must be calculated directly. Numerical integration methods to evaluate these interactions and the corresponding matrix elements affect the solution accuracy. For example, adaptive integration methods can be used to guarantee a given error ceiling for all near-field interactions. Calculating those interactions less accurately accelerates the setup stage but clearly reduces the overall accuracy.

4) Factorized Interactions: Most of the interactions (matrix elements) in MLFMA are calculated via factorization since this is necessary to reduce the complexity. But, the accuracy should not be sacrificed due to the factorization. There are well-known error controlling techniques [2] based on the worst-case analysis to determine a set of harmonics for factorized elements. A very common (but questionable) strategy is to relax these formulas and reduce the number of harmonics, which immediately decreases the solution time. The resulting implementations can be useful to develop rigorous preconditioners [11]; but they may not be reliable to produce accurate and error-controllable final results.

5) Interpolations: Multilevel structure of MLFMA requires interpolation techniques to up-sample and down-sample harmonics whenever necessary. If local interpolation methods are used, the stencil size is an important parameter for the

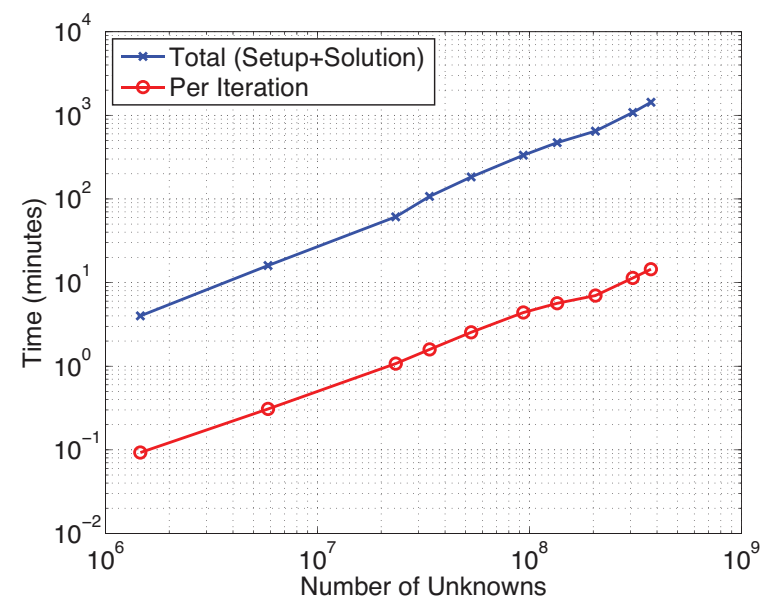

Fig. 1. The total computing time and the time per iteration for 128-process solutions of benchmark problems involving conducting spheres of radii $20 \lambda-$ $280 \lambda$ discretized with 1,462,854-374,490,624 unknowns.

accuracy and efficiency of solutions. Specifically, using fewer interpolation points reduces the computing time (due to fewer operations, and indirectly, due to easier parallelization), but deteriorates the accuracy.

6) Iterative Solver: Fast solvers, including MLFMA, are usually based on iterative solutions, where the matrix equation is satisfied approximately. The residual error of the iterative solution is another important parameter for the accuracy, as well as for the efficiency. Using higher threshold values simply reduces the number of iterations and the computing time. In addition, fewer iterations means shorter solution time compared to the setup time; this may appear to "improve" the parallelization efficiency. However, as with the other error sources, relaxing the residual threshold may significantly reduce the accuracy.

In order to measure the accuracy of solutions, we consider the far-zone scattered electromagnetic fields. For benchmark problems involving perfectly conducting spheres, scattered fields can also be obtained by Mie-series solutions, which can be used as reference results to compute the error in numerical solutions.

\section{NumericAl RESUlts}

For benchmarking purposes, we consider 11 different perfectly conducting spheres of various sizes. Specifically, we consider spheres of radii $20 \lambda, 40 \lambda, 60 \lambda, 80 \lambda, 96 \lambda, 120 \lambda, 160 \lambda$, $180 \lambda, 210 \lambda, 260 \lambda, 280 \lambda$, and $340 \lambda$. All spheres are located at the origin and illuminated by a plane wave propagating in the $-x$ direction with the electric field polarised in the $y$ direction. To measure the accuracy of numerical solutions, the far-zone co-polar electric field is calculated at 3601 points on the $x-y$ plane from $\phi=0$ (back-scattering direction) to $\phi=\pi$ (forward-scattering direction). Specifically, we compute

$$
f[n]=\frac{1}{a} \lim _{r \rightarrow \infty}\left\{r E_{\phi}(r, \theta=\pi / 2, \phi[n])\right\},
$$



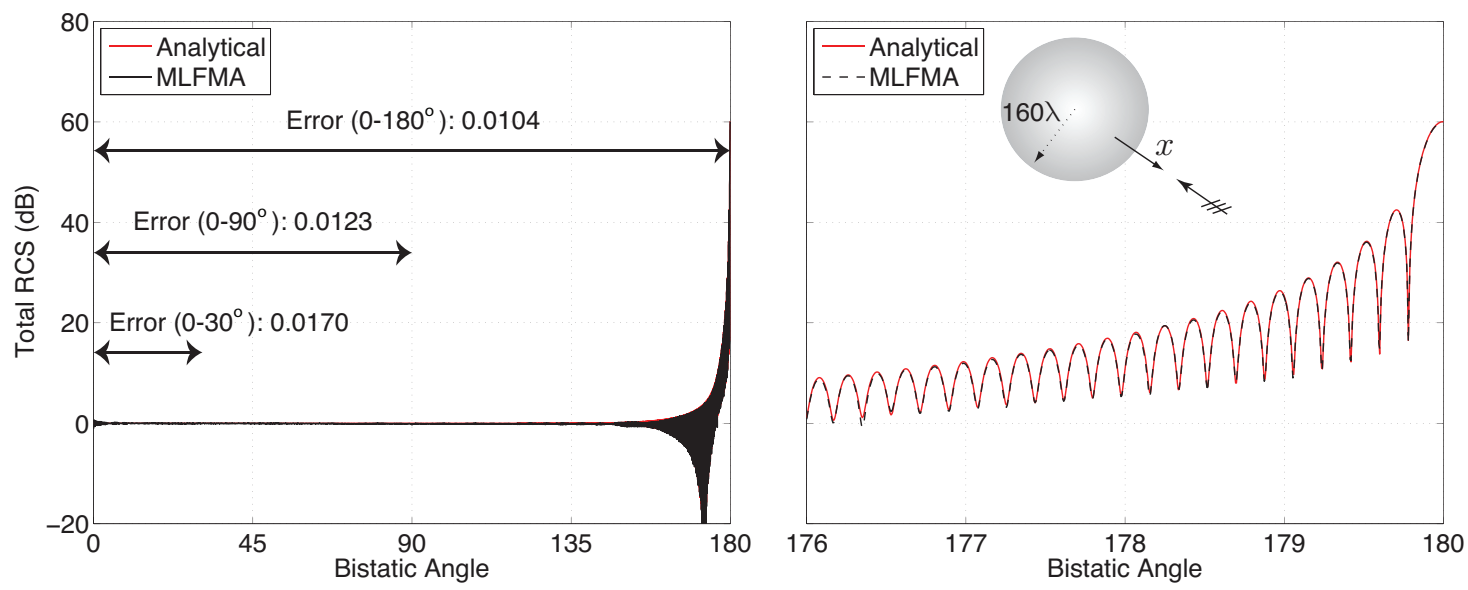

Fig. 2. Solution of a scattering problem involving a conducting sphere of radius $160 \lambda$. Normalized RCS (dB) is plotted as a function of bistatic angle from $0^{\circ}$ to $180^{\circ}$ and $176^{\circ}$ to $180^{\circ}$, where $180^{\circ}$ corresponds to the forward-scattering direction.
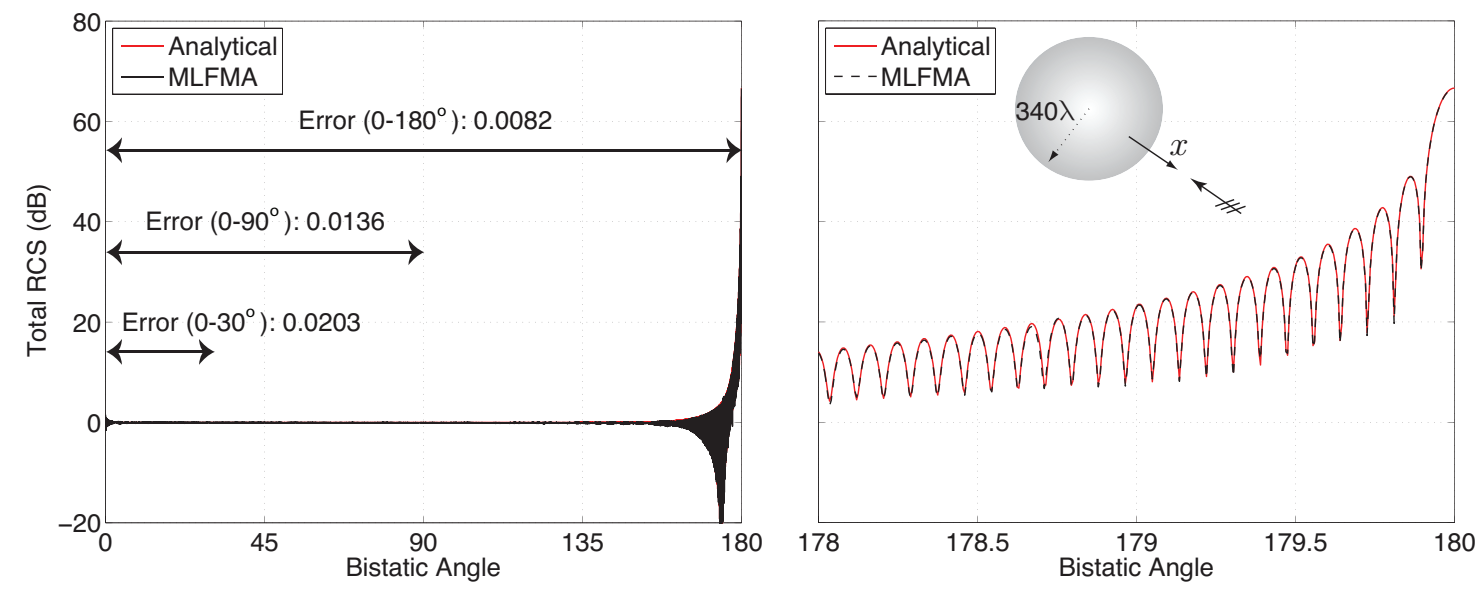

Fig. 3. Solution of a scattering problem involving a conducting sphere of radius $340 \lambda$. Normalized RCS (dB) is plotted as a function of bistatic angle from $0^{\circ}$ to $180^{\circ}$ and $178^{\circ}$ to $180^{\circ}$, where $180^{\circ}$ corresponds to the forward-scattering direction.

where $E_{\phi}$ is the $\phi$ component of the electric field and $\phi[n]=$ $(n-1) \pi / 3600$ for $n=1,2, \ldots, 3601$. In (1), the radius of the sphere in meters $(a)$ is used for normalization such that $f$ depends on the electrical size of the sphere instead of its metric size. After $f$ is calculated computationally and analytically using a Mie-series solution, the relative error is calculated as

$$
\epsilon=\frac{\left\|f_{\text {computational }}-f_{\text {analytical }}\right\|_{2}}{\left\|f_{\text {analytical }}\right\|_{2}},
$$

where $\|\cdot\|_{2}$ represents the 2-norm. One can also compute the error in different ranges by changing the boundaries, e.g, $[0, \pi / 6]$ to focus around the back-scattering direction. The relative error (2) is calculated at the interactive website www.cem.bilkent.edu.tr/benchmark when computational solutions are uploaded appropriately in the required format.

Fig. 1 presents the computing time required for the solution of the benchmark problems using a parallel MLFMA implementation on a cluster of Intel Xeon Nehalem L7555 processors with $1.87 \mathrm{GHz}$ clock rate. Solutions are parallelized into 128 processes. The total processing time (including setup and iterative solution stages) as well as the time per iteration are depicted with respect to the number of unknowns, which changes from $1,462,854$ to $374,490,624$ as the radius changes from $20 \lambda$ to $280 \lambda$. For all solutions,

- problems are formulated with $\mathrm{CFIE}=0.5 \times \mathrm{EFIE}+0.5 \times$ MFIE and discretized with the RWG functions on $\lambda / 10$ triangles,

- near-field and far-field interactions are calculated with maximum $1 \%$ error,

- interpolations are performed with $6 \times 6$ stencils, and

- and iterative solutions are carried out until the relative residual error is reduced to below 0.001 .

We observe that the smallest problem discretized with $1,462,854$ unknowns is solved in 4 minutes, whereas the largest problem discretized with $374,490,624$ unknowns is solved in less than 24 hours.

Figs. 2 and 3 present the bistatic radar cross section (RCS) 
values on the $x-y$ plane for the spheres of radii $160 \lambda$ and $340 \lambda$ discretized with 93,622,656 and 540,659,712 unknowns, respectively. After calculating $f$ in (1), the normalized RCS (dimensionless) is obtained as

$$
\operatorname{RCS}[n]=(4 \pi / \pi)|f[n]|^{2}=4|f[n]|^{2},
$$

with respect to $\phi[n]=(n-1) \pi / 3600$ for $n=1,2, \ldots, 3601$. Figs. 2 and 3 show that computational results obtained with parallel MLFMA agree well with the analytical results. In addition to RCS, the relative error in the far-zone electric field is calculated as defined in (2) from $0^{\circ}$ to $30^{\circ}, 0^{\circ}$ to $90^{\circ}$, and $0^{\circ}$ to $180^{\circ}$. Error values in these ranges are indicated in Figs. 2 and 3 . We observe that the error is around $1-2 \%$ for all ranges, indicating the high accuracy of numerical solutions.

Finally, we demonstrate the capability of our solver on a scattering problem involving a complicated airborne target, namely, the Flamme [13]. The object is investigated at $820 \mathrm{GHz}$ and its size corresponds to $1640 \lambda$. RCS values are obtained with the accuracy parameters defined above (for benchmark problems) and $\lambda / 10$ discretization leading to $538,967,040$ unknowns. The target is illuminated by a plane wave propagating at $30^{\circ}$ angle from the nose. Fig. 4 presents the co-polar (HH) and cross-polar (HV) bistatic RCS values in $\mathrm{dB}$ meter square (dBms). In addition to the forward-scattering direction $\left(210^{\circ}\right)$, we observe high RCS values at some other angles due to specular reflections. We also observe that RCS values are very low around the back-scattering direction $\left(30^{\circ}\right)$ due to the stealth property of the target.

\section{CONCLUSION}

Comparison of fast algorithms and their implementations for large-scale problems is a difficult task. For this purpose, we determine a set of benchmark problems involving perfectly conducting spheres. Reference solutions are made available on an interactive website at www.cem.bilkent.edu.tr/benchmark to evaluate the accuracy and efficiency of fast solvers. This paper presents some of the benchmark solutions obtained by using a parallel implementation MLFMA.

\section{ACKNOWLEDGMENT}

This work was supported by the Centre for Numerical Algorithms and Intelligent Software (EPSRC EP/G036136/1), the Scientific and Technical Research Council of Turkey (TUBITAK) under the Research Grant 110E268, by the Turkish Academy of Sciences in the framework of the Young Scientist Award Program (LG/TUBA-GEBIP/2002-1-12), and by contracts from ASELSAN and SSM. Özgür Ergül was also supported by a Research Starter Grant provided by the Faculty of Science at the University of Strathclyde. Computer time was provided in part by a generous allocation from Intel Corporation.

\section{REFERENCES}

[1] J. Song, C.-C. Lu, and W. C. Chew, "Multilevel fast multipole algorithm for electromagnetic scattering by large complex objects," IEEE Trans. Antennas Propag., vol. 45, no. 10, pp. 1488-1493, Oct. 1997.
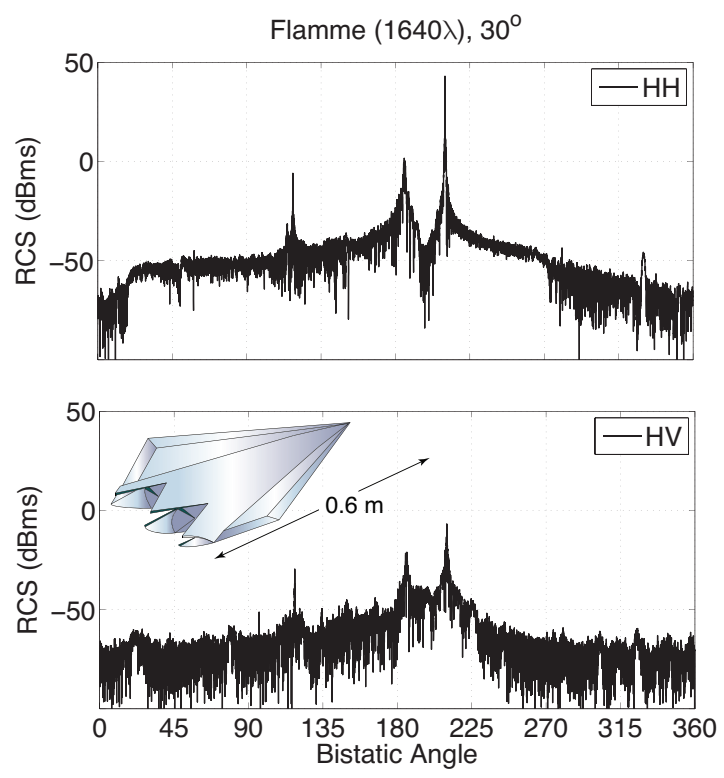

Fig. 4. Co-polar and cross-polar bistatic RCS (dBms) of the Flamme at $820 \mathrm{GHz}$. The target is illuminated by a plane wave propagating at $30^{\circ}$ angle from the nose.

[2] W. C. Chew, J.-M. Jin, E. Michielssen, and J. Song, Fast and Efficient Algorithms in Computational Electromagnetics. Boston, MA: Artech House, 2001.

[3] S. Velamparambil, W. C. Chew, and J. Song, "10 million unknowns: Is it that big?," IEEE Antennas Propag. Mag., vol. 45, no. 2, pp. 43-58, Apr. 2003.

[4] L. Gürel and Ö. Ergül, "Fast and accurate solutions of integral-equation formulations discretised with tens of millions of unknowns," Electron. Lett., vol. 43, no. 9, pp. 499-500, Apr. 2007.

[5] X.-M. Pan and X.-Q. Sheng, "A sophisticated parallel MLFMA for scattering by extremely large targets," IEEE Antennas Propag. Mag., vol. 50, no. 3, pp. 129-138, Jun. 2008.

[6] Ö. Ergül and L. Gürel, "Efficient parallelization of the multilevel fast multipole algorithm for the solution of large-scale scattering problems," IEEE Trans. Antennas Propag., vol. 56, no. 8, pp. 2335-2345, Aug. 2008.

[7] J. Fostier and F. Olyslager, "An asynchronous parallel MLFMA for scattering at multiple dielectric objects," IEEE Trans. Antennas Propag., vol. 56, no. 8, pp. 2346-2355, Aug. 2008.

[8] Ö. Ergül and L. Gürel, "A hierarchical partitioning strategy for an efficient parallelization of the multilevel fast multipole algorithm," IEEE Trans. Antennas Propag., vol. 57, no. 6, pp. 1740-1750, Jun. 2009.

[9] J. M. Taboada, L. Landesa, F. Obelleiro, J. L. Rodriguez, J. M. Bertolo, M. G. Araujo, J. C. Mourino, and A. Gomez, "High scalability FMMFFT electromagnetic solver for supercomputer systems," IEEE Antennas Propag. Mag., vol. 51, no. 6, pp. 21-28, Dec. 2009.

[10] J. M. Taboada, M. G. Araujo, J. M. Bertolo, L. Landesa, F. Obelleiro, and J. L. Rodriguez, "MLFMA-FFT parallel algorithm for the solution of large-scale problems in electromagnetics," Prog. Electromagn. Res., vol. 105, pp. 15-30, 2010.

[11] Ö. Ergül, T. Malas, and L. Gürel, "Solutions of large-scale electromagnetics problems using an iterative inner-outer scheme with ordinary and approximate multilevel fast multipole algorithms," Prog. Electromagn. Res., vol. 106, pp. 203-223, 2010.

[12] Ö. Ergül and L. Gürel, "Rigorous solutions of electromagnetics problems involving hundreds of millions of unknowns," IEEE Antennas Propag. Mag., accepted for publication, 2011.

[13] L. Gürel, H. Bağcı, J. C. Castelli, A. Cheraly, and F. Tardivel, "Validation through comparison: measurement and calculation of the bistatic radar cross section (BRCS) of a stealth target," Radio Sci., vol. 38, no. 3, Jun. 2003. 